\title{
Die zukünftigen Berufsbilder von Ärztinnen/Ärzten und Pflegenden in der ambulanten und klinischen Praxis
}

\section{Arbeitsgruppe «Berufsbilder»* der SAMW}

\footnotetext{
* Folgende Persönlichkeiten gehören der Arbeitsgruppe an, die diesen Bericht im Auftrag der Steuerungsgruppe «Zukunft Medizin Schweiz» erstellt hat: Dr. Werner Bauer, Küsnacht (Vorsitz); Prof. Anne-Françoise Allaz, Genf; Prof. Charles Bader, Genf; Barbara Gassmann, Bern; lic. oec. Pius Gyger, Zürich; Dr. Jacques de Haller, Bern; Dr. Romy Mahrer Imhof, Basel; Dr. Peter Tschudi, Basel; Priska Wildhaber Isler, Küsnacht.
}

** Aus Gründen der leichteren Lesbarkeit wird in diesem Text gelegentlich nur die männliche Bezeichnung verwendet; selbstverständlich gilt sie jeweils für beide Geschlechter.

Korrespondenz:

SAMW

Petersplatz 13

CH-4051 Basel

mail@samw.ch

\begin{abstract}
Auftrag und Vorgehen der Arbeitsgruppe Der Bericht «Ziele und Aufgaben der Medizin zu Beginn des 21. Jahrhunderts» weist darauf hin, dass sich das Berufsbild des Arztes** und der Pflegefachpersonen in den vergangenen Jahrzehnten radikal gewandelt hat; dies habe gleichzeitig auch dazu geführt, dass sich Ärzte und Pflegende in ihrer Berufsidentität verunsichert fühlen. Der Bericht geht davon aus, dass es in Zukunft zu weitgehenden Veränderungen der Rollendefinitionen unter den Berufen im Gesundheitswesen kommen wird.

Die Steuerungsgruppe des Projektes «Zukunft Medizin Schweiz» hat dieser Problematik eine hohe Bedeutung zugemessen und deshalb beschlossen, sie zu einem Schwerpunkt der Phase III zu machen und eine Arbeitsgruppe mit folgendem Auftrag einzusetzen:
\end{abstract}

Die Arbeitsgruppe beschreibt in einem ersten Schritt die aktuellen Rollendefinitionen (Innen- und Aussensicht) der verschiedenen Berufe im Gesundheitswesen (Ärztinnen/Ärzte und Pflegefachpersonen), skizziert die wahrscheinlichen Entwicklungslinien, benennt die möglichen Konfliktfelder und versucht, einen sinnvollen Soll-Zustand zu definieren.

In einem zweiten Schritt hält die Arbeitsgruppe fest, welche Massnahmen im Ausbildungsbereich und allenfalls in anderen Bereichen (z.B. neue Rollenverteilung; Öffentlichkeitsarbeit) getroffen werden müssten, um die Konfliktfelder zu entschärfen und allenfalls dem Soll-Zustand näher zu kommen.

Dieser Auftrag ist komplex und setzt ein hohes Ziel. Die Arbeitsgruppe ist sich bewusst, dass es bei der Bearbeitung dieser Fragestellung zahlreiche Abgrenzungs- und Schnittstellenprobleme mit anderen Berufen (z.B. Fachangestellte Gesundheit, medizinische Praxisassistentin) gibt. Da die Beziehung zwischen den diplomierten Pflegefachpersonen und den Ärztinnen und Ärzten als exemplarisch angesehen werden kann und hier auch zahlreiche Konfliktfelder zu orten sind, hat sich die Arbeitsgruppe bei ihrer Analyse und ihren Empfehlungen auftragsgemäss auf diese beiden Berufsgruppen beschränken müssen. Sie hat sich dabei immer am Machbaren orientiert und versucht, den Bezug zur Realität nicht zu verlieren. Ins Zentrum ihrer Überlegungen hat die Arbeitsgruppe die Bedürfnisse der einzelnen Patienten gestellt, zudem hat sie sich an den Voraussetzungen für eine optimale Gesundheitsversorgung der Bevölkerung orientiert.

Der Text, der nun vorliegt, ist als Diskussionsgrundlage zu betrachten. Die Kenntnis des von der SAMW publizierten Berichtes «Ziele und Aufgaben der Medizin zu Beginn des 21. Jahrhunderts» muss als Grundlage vorausgesetzt werden. Es liegt in der Natur der Sache, dass die Einschätzungen und Gewichtungen der Entwicklungen und ihrer Konsequenzen innerhalb der Arbeitsgruppe nicht absolut deckungsgleich sein können. Meinungsunterschiede bestehen durchaus; weil der Text aber eine Konsensarbeit der Gruppe darstellt, erachten es deren Mitglieder nicht als notwendig, diese Meinungsunterschiede explizit aufzuzeigen.

Die Arbeitsgruppe versteht unter dem Begriff des Berufsbildes die Beschreibung der Tätigkeiten und Aufgaben einer Berufsgruppe sowie die Darstellung des Umfeldes und der grundlegenden Voraussetzungen (gesellschaftliches Umfeld, Arbeitsbedingungen, Aus- und Weiterbildung) zur Ausübung des betreffenden Berufes. Ein Berufsbild enthält keinen detaillierten Lernzielkatalog.

Unter dem Begriff der Berufsidentität versteht die Arbeitsgruppe das Spezifische, das die Ausübenden eines Berufes für ihn empfinden, was den Beruf charakterisiert und ihn unterscheidet. Der folgende Text geht nicht speziell auf die Differenzierung dieser Begriffe ein.

Die Arbeitsgruppe versteht ihr Dokument als Grundlage für eine breite Diskussion, und sie hofft, dass diese breite Diskussion im gleichen offenen, konstruktiven, zukunftsgerichteten Geist stattfinden wird, wie er innerhalb der Gruppe geherrscht hat. 
Die Medizin im gesellschaftlichen Kontext Ärzte und Pflegefachleute arbeiten nicht in geschützten Räumen. Die gesellschaftlichen Entwicklungen in allen Bereichen (vom Werte- bis zum Wirtschaftssystem) haben entscheidenden Einfluss auf die Rahmenbedingungen ihrer Tätigkeit. Die folgenden Trends sind von besonderer Bedeutung:

\section{Zunehmende Diversifizierung}

Das Gesundheitswesen wandelt sich schnell und wird immer diversifizierter: mehr Anbieter, konkurrierende Modelle und Organisationen, neue Formen der Leistungserbringung, eine Vielfalt von Methoden, neue Tarif- und Lenkungssysteme. Ebenso wird die freie Arztwahl in Frage gestellt. Die Politik beeinflusst zunehmend alle Ebenen des Gesundheitswesens - und damit auch die Berufsbilder. Auch die Versicherer sind zu wichtigen Akteuren geworden, die ihre Interessen offensiv vertreten.

Wir beobachten eine zunehmende Multikulturalität sowohl bei den Patienten als auch bei den Mitarbeitenden.

Was bleiben wird, ist aber das Essentielle: Es wird immer Leidende geben, die Hilfe suchen, und die Kernaufgabe des Gesundheitswesens wird es immer sein, sich der Leidenden anzunehmen und ihnen diese Hilfe nach besten Kräften zu vermitteln.

\section{Von der Gesundheitsversorgung zum «Gesundheitsmarkt»}

Die Spannungen zwischen dem auf den Menschen und auf das Menschliche zentrierten Auftrag des Gesundheitswesens und den immer mehr dominierenden ökonomischen Faktoren nehmen weiter zu. Die Entwicklungen im Gesundheitswesen werden zunehmend von wirtschaftlichen Interessen, den Marktkräften und ökonomischen Zwängen beeinflusst.

Auf der einen Seite erwirtschaften erfolgreiche Firmen (von Spital- und Laborketten über die pharmazeutische Industrie bis zur Medizintechnologie) hohe Gewinne, auf der anderen Seite bestehen drückende finanzielle Zwänge, die weitherum zu Ratlosigkeit führen und die Hoffnung aufkeimen lassen, dass sich in und hinter dem, was das Wort «Management» beinhaltet, die Lösung der Probleme verbergen könnte.

Eine Anspruchshaltung mit entsprechenden Forderungen an das Gesundheitswesen, dessen Leistungen mehr und mehr als Konsumgut betrachtet werden, nimmt zu. Die Erwartung, dass alle Massnahmen sofort, perfekt und erfolgreich zu geschehen haben, steigt unter anderem auch in Relation zu den steigenden Versicherungsprä- mien. Gleichzeitig verringern sich zusehends die Basiskompetenzen der Bevölkerung in Gesundheitsfragen, und die Fähigkeit, kleinere Gesundheitsstörungen selbst zu bewältigen, geht zunehmend verloren. Zudem wird der Trend anhalten, wirtschaftliche und soziale Probleme zu medikalisieren.

\section{Feminisierung}

Während die Pflege schon lange als «klassischer Frauenberuf» gilt, zeichnet sich in der Medizin seit einiger Zeit eine ähnliche Entwicklung ab. Der Anteil der Frauen im Medizinstudium beträgt über $60 \%$, und er ist weiter am Steigen. Diese «Feminisierung» wird unweigerlich Auswirkungen auf das Berufsbild haben.

\section{Informationsflut}

Die zur Verfügung stehenden Informationen kommen immer mehr in Form einer papierenen und elektronischen Informationsflut daher, was die Orientierung für die Laien erschwert oder sogar verunmöglicht.

Um die heute angestrebten Ziele im Sinne von «shared decision making» oder «patient empowerment» zu erreichen, braucht es die Gewichtung der Information durch eine medizinische Fachperson.

\section{Die Veränderungen der beruflichen Tätigkeit}

Immer deutlicher beobachten wir auch Veränderungen in der Ausübung der ärztlichen und pflegerischen Berufe. Allerdings gibt es neben den Kräften des Wandels auch bremsende Elemente, was in der Schweiz gegenwärtig das mosaikartige Bild eines Nebeneinanders von sehr traditioneller Leistungserbringung und zahlreichen Pilotprojekten und -modellen ergibt.

\section{Neue Berufe tauchen auf}

Die wohlbekannten und vermeintlich klardefinierten Berufsbilder von Hausärzten, Spezialärzten, Pflegenden und anderen Berufsleuten im Gesundheitswesen geraten ins Rutschen. Neue Berufe tauchen auf, die Grenzen zwischen den bestehenden verwischen sich zum Teil. Erste Ansprechperson im Gesundheitswesen und Anbieter verschiedener Untersuchungen und Therapien ist nicht mehr notwendigerweise eine Ärztin oder ein Arzt, wie dies bisher mit Ausnahme der Alternativmedizin weitgehend die Regel war.

\section{Vernetzung}

Teamarbeit und Vernetzung werden allgemein als Modelle der Zukunft betrachtet. Allerdings wird es in unserem Land noch lange Einzelpraxen, kleine Gruppenpraxen und auch entsprechende 
Organisationsformen im spitalexternen Bereich der Pflege geben. Der sinnvolle Wunsch nach einer langjährigen persönlichen Beziehung zu einer Hausärztin oder einem Hausarzt ist in unserer Bevölkerung noch sehr stark verwurzelt, wobei sich viele, vor allem auch jüngere Leute, durchaus die Freiheit nehmen wollen, von Fall zu Fall zu entscheiden, wohin sie sich wenden.

\section{Aus- und Weiterbildung im Wandel}

Auch Aus- und Weiterbildung sind einem Wandel unterworfen, der mit der Einführung des Bologna-Modells im Bereich der Ärzte- und Pflegeausbildung zum ersten Mal eindrücklich manifest geworden ist. Die Konsequenzen sind noch nicht klar absehbar, und Veränderungen auch in der Strukturierung der Weiterbildung werden folgen.

\section{Die Arbeitsbedingungen ändern sich}

Von Ärzten und Pflegenden werden im Normalbetrieb keine Arbeitszeiten mehr verlangt werden können, die über diejenigen vergleichbarer Berufe (mit Notfall-, Nacht- und Feiertagsdienst) und Kaderfunktionen hinausgehen.

Konfliktfelder verschiedenen Grades und verschiedener Art bestehen schon im Bereich folgender Schnittstellen, und sie öffnen sich immer weiter:

- Spezialärzte $><$ Ärzte in der ärztlichen Grundversorgung;

- Ärzte $><$ Pflegende $><$ andere Berufe im und um das Gesundheitswesen;

- selbständige Ärzte und Pflegende $><$ Spitäler, Heime, Spitex-Organisationen;

- Medizin >< Ökonomie;

- Leistungserbringer $><$ Kostenträger.

\section{Leitgedanken zu einer Medizin der Zukunft}

Die Berufsbilder der Medizinalberufe erfahren Veränderungen und werden weitere Veränderungen erfahren, die den Beteiligten erst nach und nach bewusst werden. Für die junge Generation, die ihre Berufswahl und Karriere plant, sind die Berufsbilder und die auf ihnen basierenden beruflichen Perspektiven entscheidend wichtig. Mit Hilfe einer strukturierten Aus- und Weiterbildung sowie begleitenden Massnahmen muss versucht werden, einen definierten essentiellen Kern von Haltungen, Kenntnissen und Fähigkeiten, die ein Berufsbild prägen, zu erhalten oder auszubauen.

\section{Niederschwellige Anlaufstelle}

Die erste Ansprechperson im Gesundheitswesen ist nicht notwendigerweise eine Ärztin oder ein Arzt.
Die erste Anlaufstelle soll eine niederschwellig kontaktierbare Institution (Praxisgemeinschaft, Zentrum, «medical home», Notfallstation, Netzwerk) sein, wo alle nichtspezialisierten Abklärungen und Behandlungen durchgeführt werden können und von wo direkte Überweisungen an die kompetenten Stellen erfolgen. Mit klaren Einschränkungen können auch Callcenter, Internetportale und Apotheken die Funktion einer ersten Anlaufstelle übernehmen.

\section{Interdisziplinäre Teamarbeit}

Die Aufgabenverteilung wird sich in der Zukunft nicht mehr an hierarchischen, ständisch definierten oder mit einem formellen Titel verbundenen Kriterien orientieren. Interdisziplinäre Teamarbeit gewinnt mehr und mehr an Bedeutung: Ärzte werden vor allem im Bereich der Diagnose- und Indikationsstellung, Behandlung und Beratung, des «Disease Management» komplexer Fälle und der spezialisierten Methoden ihre Wirkungsfelder haben. Pflegende werden zusätzliche Funktionen in Diagnostik, Therapie und Case Management übernehmen und diese selbstverantwortlich ausführen. Bestimmte technische Methoden, wie zum Beispiel die Sonographie, diagnostische Endoskopien oder Laseranwendungen, werden wahrscheinlich mehr und mehr auch von Nichtärzten oder von Ärzten durchgeführt werden, die eine selektive Weiterbildung durchlaufen haben. Eine solche könnte allenfalls auch zu einer anderen Zertifikation als einem Facharztdiplom führen.

Je weiter sich die Spezialisierung entwickelt und verästelt, desto wichtiger werden Generalisten mit immer wieder aktualisierten Kenntnissen der Methoden und Technologien, die zur Verfügung stehen. Ihre Aufgabe ist es, die Wegmarken entlang den Diagnostik- und Behandlungspfaden zu setzen.

\section{Orientierung an den Bedürfnissen der Patienten}

Die Berufsbilder müssten sich idealerweise an den Bedürfnissen der Patientinnen und Patienten orientieren; starke andere wirtschaftliche und politische Interessengruppen werden aber ihren Einfluss geltend machen. Dieser Einfluss wird sich vor allem über die Finanzierung auswirken und damit auch Konsequenzen bei der Finanzierung von Ausund Weiterbildung haben. Die Weiterbildung wird mehr und mehr mit der Forderung konfrontiert, strukturiert und von einem möglichst frühen Zeitpunkt an gezielt zu erfolgen. Diese Forderung könnte auch auf die Ausbildungsphase ausgedehnt werden; das Bologna-Modell bietet die dafür nötigen Instrumente an sich bereits an. 


\section{Lebenslanges Lernen}

Die Weiterbildung muss strukturiert und modular aufgebaut sein.

Das Spektrum der Tätigkeiten zu Beginn der Berufsarbeit wird immer seltener identisch sein mit demjenigen zum Zeitpunkt der Pensionierung. Deshalb muss es auch im Laufe eines Berufslebens mit vertretbarem Aufwand möglich sein, sich neue Kompetenzen anzueignen und das Berufsfeld zu wechseln.

Zertifikaterteilung und Selektionsprozesse sind sinnvolle Instrumente zur Überprüfung von Kenntnissen und Fähigkeiten; gleichzeitig bergen sie die Gefahr, unter dem Vorwand der Qualitätssicherung von Interessengruppen für die Monopolisierung oder für die Zugangssteuerung missbraucht zu werden.

Lebenslange, gezielte Fortbildung und die Bereitschaft zur stetigen Sicherung und Förderung der Qualität sind selbstverständliche Voraussetzungen zur Ausübung der Berufe im Gesundheitswesen. Die Überprüfung von Fortbildung und Qualität sowie das Erarbeiten von Qualitätskontrollinstrumenten müssen auf evidenzbasiertem Konsens beruhen und können nicht einseitig Sache der Leistungserbringer, einer staatlichen Stelle oder der Versicherer sein.

Diagnostizieren und Behandeln sind nicht alles Ein aktives Engagement in Prävention und Gesundheitsförderung, Lehre und Forschung, ein
Interesse für «Public Health»-Aspekte sowie ökonomische Grundkenntnisse gehören ins Berufsbild der Gesundheitsberufe.

\section{Das Berufsbild der Ärztinnen/Ärzte und Pflegefachpersonen}

Das Berufsbild der Ärzte und Pflegenden lässt sich dreistufig beschreiben und besteht aus (Abb. 1):

1. den gemeinsamen Grundelementen der Berufe des Gesundheitswesens:

- das Basiswissen um das Wesen des Menschen und seine Funktionen;

- die ethischen und deontologischen Prinzipien und eine darauf basierende Grundhaltung im Sinne der «medical humanities»;

- grundlegende praktische Fertigkeiten;

- die gemeinsame Sprache und die Fähigkeit, mit Patienten und anderen Fachpersonen adäquat zu kommunizieren;

- Grundkenntnisse des schweizerischen Gesundheitswesens, der rechtlichen Grundlagen und der wirtschaftlichen Zusammenhänge;

- Basiskenntnisse der Qualitätssicherung;

- Verständnis für Lehre und Forschung.

Die Vermittlung dieser Kenntnisse wird in die Ausbildungsgänge der jeweiligen Berufsgruppen integriert; gemeinsame Module sind vorstellbar.

2. den gruppenspezifischen Berufsbildelementen je aller Ärzte und aller Pflegenden (spezifische, aber

\section{Abbildung 1}

Die Berufsbilder der Ärzte und Pflegenden lassen sich dreistufig beschreiben: 1. Grundelemente, die allen Berufen des Gesundheitswesens gemeinsam sind (z. B. ethische Prinzipien oder die Fähigkeit, mit Patienten zu kommunizieren); 2. berufsspezifische Elemente sowie 3. spezialisierte Elemente.

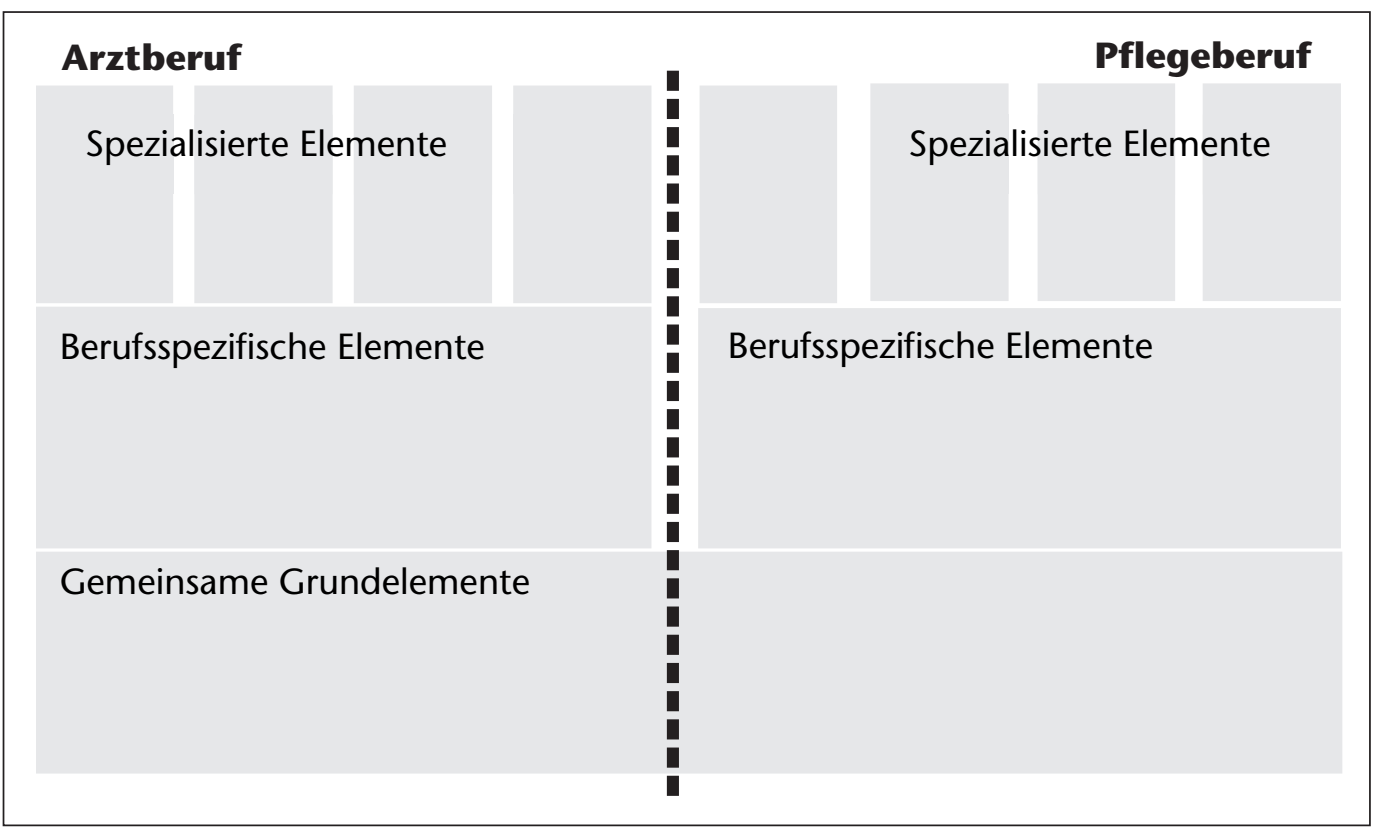


nicht spezialisierte Kenntnisse der normalen und gestörten Funktionen des Menschen sowie die nötigen Grundfähigkeiten und -fertigkeiten wie Gesprächsführung, Beobachtung, Basisuntersuchungs- und Behandlungstechniken)

3. den besonderen Elementen der einzelnen Berufe im ärztlichen und pflegerischen Bereich (spezialisierte, technische oder andere Kompetenzen je nach Funktion)

\section{Das zukünftige Berufsbild der Ärztinnen und Ärzte}

\section{Aufgaben und Tätigkeiten}

Der Arztberuf ist ein zentraler Beruf im schweizerischen Gesundheitswesen mit dem Auftrag der Prävention, der Diagnostik und der Therapie akuter Gesundheitsstörungen, der Palliation und der Rehabilitation sowie der Langzeitbetreuung bei chronischen Leiden im Verbund mit anderen Berufsgruppen. Grundelement bleibt die persönliche, oft langfristige zwischenmenschliche Beziehung von Arzt und Patient.

Der Beruf umfasst zwei Haupttätigkeitsfelder, dasjenige des in der medizinischen Grundversorgung tätigen Haus-, Heim- oder Spitalarztes und dasjenige des Spezialisten, der eine bestimmte Methode oder Technik beherrscht und sie im Rahmen eines Diagnostik- oder Behandlungskonzepts bei einem bestimmten Patienten anwendet. Die Zweiteilung der Medizin in einen ambulanten und einen stationären Sektor wird weiterhin bestehen, ändert jedoch zunehmend ihren Charakter (Wachstum des ambulanten Sektors, kürzere Hospitalisationen, personelle Verflechtung).

Beide Ärztegruppen üben ihre Tätigkeit in enger Zusammenarbeit und in einzelnen Bereichen auch im Wettbewerb mit anderen Berufsleuten aus.

Diese Ärztinnen und Ärzte, die in der ambulanten (Hausarztmedizin) und in der stationären (Notfallstationen und Abteilungen und Kliniken für allgemeine Innere Medizin) ärztlichen Grundversorgung tätig sind, haben in der Regel eine Weiterbildung in Allgemein- oder Innerer Medizin (in Zukunft im Hinblick auf die europäische Anerkennung ein Facharzttitel, der die international gültigen Kriterien für die allgemeine Innere Medizin erfüllt) hinter sich, oder sie sind Kinderärzte.

Auch innerhalb vieler Spezialfächer gibt es wiederum eine Unterteilung in Grundversorgung und hochspezialisierte Medizin (z.B. praktizierende Augenärzte und Glaskörper- oder Netzhautchirurgen).
Die generalistisch tätigen Ärztinnen und Ärzte sind Experten in der Gesprächsführung (Anamnese, «shared decision making», Beratung), in der klinischen Diagnostik und Therapie, in der prioritätensetzenden Beurteilung komplexer gesundheitlicher Probleme, in der Koordination diagnostischer und therapeutischer Methoden und in der Indikationsstellung im Hinblick auf die Überweisung an Spezialisten und spezialisierte Zentren.

Je nach Arbeitsplatz und persönlicher Kompetenz führen sie die indizierten technischen Massnahmen (z.B. Ultraschalluntersuchungen, kleine diagnostische und kurative Eingriffe) selbst durch, oder sie überweisen die Patienten für alle Massnahmen, die effizienter, wirtschaftlicher oder kompetenter durch eine andere Fachperson durchgeführt werden können, an eine solche Fachperson innerhalb oder ausserhalb ihres eigenen Teams.

Sie beteiligen sich am Notfalldienst und besuchen ihre Patienten zu Hause oder in Pflegeinstitutionen. Die Koordination, die Interpretation von Befunden, das Erarbeiten von Konsequenzen und die Kommunikation mit den Patienten sind ihre Kernaufgaben.

Die Attraktivität des Berufes beruht auf der fachlichen Herausforderung bei der Bewältigung komplexer Probleme, auf den oft lange dauernden, zwischenmenschlichen Beziehungen mit den Patienten und im Team und auf der Schlüsselstellung, die diese ärztliche Tätigkeit aufgrund der Entscheidungskompetenzen im Gesundheitswesen einnimmt.

Die Spezialärztinnen und -ärzte sind Fachleute mit höchster Kompetenz in einem bestimmten methodischen Bereich. Dabei kann es sich um technische Anwendungen (z. B. spezialisierte chirurgische Eingriffe, invasive diagnostische Verfahren, radio-onkologische Therapien) oder um andere Spezialverfahren (z. B. Psychotherapien und onkologische Behandlungsmethoden) handeln.

Bei Patienten mit einem einzigen wesentlichen Krankheitsbild kann der Spezialist für die Zeitperiode der spezialärztlichen Behandlung auch die integrale ärztliche Verantwortung übernehmen. In allen Fällen, wo mehrere gesundheitliche und allenfalls auch zusätzliche soziale, psychosoziale, pflegerische oder andere Probleme bestehen, erfolgen die spezialärztlichen Massnahmen in enger Zusammenarbeit mit dem hausärztlichen Betreuungsteam. Die spezialärztliche Tätigkeit erfordert die volle Kompetenz für das betreffende Spezialfach, dessen Leistungen aber immer im Rahmen eines umfassenden, kurativen oder 
palliativen Behandlungs- und Betreuungskonzeptes erbracht werden müssen.

Die Attraktivität des Berufes beruht auf der fachlichen Herausforderung durch die Anwendung moderner, anspruchsvoller, sich wandelnder technischer oder anderer Methoden, auf dem hochwirksamen Beitrag zur Diagnostik und Therapie im Rahmen eines Teams oder eines Netzwerks und auf dem Bewusstsein, bei korrekter Indikationsstellung den Patienten entscheidende Hilfe leisten zu können.

$\mathrm{Zu}$ allen ärztlichen Berufen gehört die Bereitschaft zu einem temporär überdurchschnittlichen Einsatz bei der Bewältigung von Notfallsituationen und zum Leisten von Nacht- und Feiertagsdienst.

\section{Berufliches Umfeld}

Ärztinnen und Ärzte können sich beruflich nur dann voll und ihrer Aus- und Weiterbildung entsprechend entfalten, wenn die Arbeits- und Rahmenbedingungen adäquat sind.

Die diagnostische und therapeutische Freiheit im Rahmen anerkannter beruflicher und fachlicher Standards (Richtlinien, WZW) bleibt - ob Arzt in freier Praxis oder im Angestelltenverhältnis - eine Grundvoraussetzung für die ärztliche Tätigkeit.

Ökonomische Faktoren, Rahmenbedingungen, Argumente bis hin zu Zwängen prägen die heutige Medizin mit. Ärzte müssen fähig sein, an ihren Arbeitsplätzen ihre Position überzeugend einzubringen und wirkungsvoll zu vertreten. Dies gilt im Hinblick auf die Kosten verordneter Massnahmen, doch auch das korrekte Wahrnehmen eigener Interessen steht jedem Berufsstand zu und kann immer wieder notwendig sein.

Mehr und mehr arbeiten auch die ambulant tätigen Ärztinnen und Ärzte in einem Netzwerk, das in Form eines Teams in einem Zentrum oder auch dezentralisiert organisiert sein kann. Die Zusammenarbeit mit den Pflegenden ist für die zweckmässige Betreuung der Patienten von zentraler Bedeutung, wobei diese Zusammenarbeit nicht durch ein formell hierarchisches Verhältnis geprägt ist, sondern von der Fragestellung ausgeht, wer für welche Funktion kompetent ist und sie optimal erfüllen kann. Nach erfolgter ärztlicher Abklärung und bei klarer Therapie gibt es bestimmte Beratungen, Nachkontrollen und Behandlungsmassnahmen, die im Rahmen eines definierten Konzepts sinnvollerweise und eigenverantwortlich an Pflegende übergeben werden können.

Die Integration der ärztlichen Tätigkeit in Teamarbeit muss zu einer Entlastung von administrativer Tätigkeit, von der Bewältigung des Per- sonalwesens, vom Bestellwesen und von ähnlichen paramedizinischen Pflichten führen und erlaubt damit die Konzentration auf das Wesentliche des Arztberufes.

Die Entlöhnung muss der beruflichen Qualifikation und der zu tragenden Verantwortung entsprechen und so ausgestaltet sein, dass keine unerwünschten Anreize entstehen. Interessenkonflikte in Richtung Mengenausweitung sind ebenso zu vermeiden wie solche in Richtung Minderversorgung.

\section{Voraussetzungen zur Ausübung des Berufes} Der Arztberuf hat als erste Voraussetzung den Abschluss eines Medizinstudiums auf der Masterstufe und das Bestehen des Staatsexamens. Dieser stellt die allen gemeinsamen Basiskenntnisse, die gemeinsame Sprache und, soweit möglich, eine gemeinsame Grundhaltung sicher.

$\mathrm{Ob}$ in Zukunft spezifische Masterabschlüsse für bestimmte medizinische Berufsfelder angeboten werden, ist denkbar, aber noch ungewiss. Es ist zum Beispiel vorstellbar, dass für den Bereich der Forschung oder für umschriebene, technisch orientierte Tätigkeitsfelder ein eigener Abschluss in Frage kommt, ein Weg, den ja die Zahnärzte schon lange gegangen sind. Für alle ärztlichen Funktionen, die über einen Einzeleingriff an einem bestimmten Patienten hinausgehen, ist aber der gleiche, allgemeinverbindliche Studienweg und Abschluss notwendig.

Die zweite Voraussetzung besteht in einer Weiterbildung, nach deren Abschluss eine Person befähigt sein muss, im entsprechenden Fachbereich eigenverantwortlich tätig zu sein. Die Wichtigkeit einer strukturierten, systematisch auf das gewählte Tätigkeitsfeld vorbereitenden Weiterbildung kann gar nicht genügend betont werden. Sie hat grundsätzlich für alle Facharzttitel gleichwertig zu sein.

Im Laufe der Weiterbildungszeit muss auch das Bewusstsein der persönlichen Verantwortung, die Bereitschaft zur Arbeit in einem Team sowie zu Lehre und Forschung, die Einsicht in die Notwendigkeit der Fortbildung und des Hinterfragens der Qualität gefördert werden - mithin alle Aspekte, die im englischen Sprachbereich mit dem Wort «professionalism» bezeichnet werden.

Basiskenntnisse ökonomischer Mechanismen und Zusammenhänge sind für die tägliche Arbeit und für erfolgreiches Argumentieren unabdingbar, ebenso die Bereitschaft, die eigene Arbeit zu hinterfragen, sich gezielt fortzubilden und an Qualitätssicherungsprojekten teilzunehmen.

Qualitätsmassnahmen, die von staatlichen Stellen oder von Versicherern verlangt werden, bedürfen einer unbestreitbaren Legitimation. 
Ein flexibel und modular gestaltetes Weiterbildungssystem muss es ermöglichen, sich auch im Laufe des Lebens mit vertretbarem zeitlichem Aufwand beruflich umzuorientieren und in einem benachbarten Fachbereich die nötige Kompetenz zu erwerben.

\section{Das zukünftige Berufsbild der Pflegenden}

Das Berufsbild der diplomierten Pflegefachfrau/ des diplomierten Pflegefachmanns basiert auf den gemeinsamen Grundelementen der Berufe des Gesundheitswesens und auf der Definition der professionellen Pflege, wie sie nachstehend erläutert wird.

\section{Aufgaben und Tätigkeiten}

Menschen jeden Lebensalters und in unterschiedlichsten Lebenssituationen können auf Pflegeleistungen von ausgebildeten Pflegefachpersonen angewiesen sein. In der Prävention geht es darum, Gesundheit von Individuen und Familien zu fördern und zu erhalten und gesundheitlichen Schäden vorzubeugen. Kranke Menschen brauchen Pflegeinterventionen bei der Behandlung ihrer Krankheiten. Sie und ihre Familien brauchen Unterstützung im Umgang mit Auswirkungen von Krankheiten und Therapien auf ihren Alltag. Das Tätigkeitsfeld umfasst die Durchführung von Massnahmen beim einzelnen und in der Familie zur Wiederherstellung der Gesundheit, zur Bewältigung des Krankseins und zur Begleitung in Krisen und beim Sterben. Ein weiteres Tätigkeitsfeld der Pflegenden kann eine bestimmte Zielgruppe oder die gesamte Bevölkerung darstellen. Dort geht es darum, Strategien zur Sicherstellung einer bedarfsgerechten Pflege und/oder Strategien zur Förderung der langfristigen Gesundheit zu entwickeln.

Professionelle Pflege wird in unterschiedlichsten Settings im Gesundheitswesen angeboten: gemeindenah (Spitex-Organisationen, Mütter-/ Väterberatung, Gemeinschaftspraxen, Gesundheitszentren), in hochspezialisierten Institutionen (Universitäts- und Spezialkliniken) oder in der Gesundheitsförderung/Prävention (Arbeitsplätze, Schulen).

Pflegende mit einer generalistischen Ausbildung auf Niveau Bachelor übernehmen die pflegerische Versorgung von Patientinnen und Patienten im stationären, ambulanten oder häuslichen Bereich. Sie schätzen gemeinsam mit den Patienten und ihren Angehörigen den Bedarf an Pflege und Betreuung ein, planen Massnahmen, führen diese durch und evaluieren ihre Arbeit. Sie sind Experten in Gesprächsführung (Anamnese, shared decision making, Beratung). Sie beurteilen den
Bedarf an notwendiger Unterstützung zur Durchführung von Therapien und den Unterstützungsbedarf in den Aktivitäten des täglichen Lebens. Sie beraten und begleiten Patienten und Angehörige gezielt, um das Selbstmanagement im Umgang mit Krankheit und Therapie zu erhöhen und die Adhärenz zu Therapien zu stärken. Sie beraten Patienten und Angehörige darin, gesundheitliche Risiken zu erkennen und $\mathrm{zu}$ mindern.

Pflegende haben bei der Gestaltung des Betreuungsplans eine wichtige Koordinationsfunktion. Ziel dabei ist, Auswirkungen des Krankseins auf den Alltag des Patienten möglichst gering zu halten. Pflegende planen die Betreuung in Zusammenarbeit mit ärztlichen Kollegen und unter Einbezug weiterer Berufsangehöriger (Hauspflege, Sozialdienst, Physiotherapie usw.).

Ihre Ausbildung erlaubt den Pflegenden, ihre fachlichen Grenzen zu erkennen und Fachpersonen anderer Gesundheitsberufe bei Bedarf beizuziehen (z.B. «Hausarzt», Spezialärzte, Ernährungsberaterin),

Die Optik der Pflegenden ist weniger auf die Krankheit als auf das Kranksein ausgerichtet und darauf, zu helfen, mit Unzulänglichkeiten infolge gesundheitlicher Beeinträchtigung im Alltag zurechtzukommen; ihr Denken ist durch die Nähe zum Patienten und zu seinem Alltag gekennzeichnet.

Pflegende, die sich einer vertieften klinischen Praxis zuwenden, können bezogen auf eine definierte Patientenpopulation (z.B. Herzinsuffizienz, Onkologie, Neugeborene) oder bezogen auf ein bestimmtes Versorgungssetting (Ambulatorium, Spezialklinik, Spitex, Intensivstation usw.) qualifizierte Arbeit leisten. Pflegende mit vertiefter Praxis sind auf Masterstufe ausgebildet. Sie verfügen über erweiterte Kompetenzen im technischmedizinischen Bereich und/oder in Beratung und Begleitung (z.B. spezialisierte geriatrische Pflege, pädiatrische Pflege, Diabetesberatung, Herzinsuffizienzberatung, koronare Rehabilitation). Diese Pflegenden haben eine Weiterbildung in spezialisierten Fachbereichen absolviert. Sie sind dazu ausgebildet, neue Konzepte der Versorgung in einer Institution einzuführen und andere Pflegende evidenzbasiert anzuleiten. Sie sind in der Lage, neue Konzepte der Versorgung und Beratung zu entwickeln und einzuführen. Sie wenden dazu wissenschaftliche Methoden der Erkenntnisgewinnung an.

\section{Berufliches Umfeld}

Pflegende können den Beruf im Angestelltenverhältnis oder in eigener Praxis/Pflegeinstitution 
anbieten. Ihr Handeln orientiert sich an den ethischen Normen und an den definierten Qualitätsstandards ihres Berufsstandes.

Gegenüber Dritten bringen Pflegende ihre Position bei der Begleitung von Patienten und Angehörigen überzeugend ein und vertreten diese wirkungsvoll. Sie sind in der Lage, eigene berufliche Interessen sowie die Interessen der Pflegeabhängigen zu vertreten.

Pflegende sind wesentliche Kooperationspartner in den Netzwerken der medizinischen Versorgung. Sie sind befähigt, in Teams zu arbeiten. Diese Teams können in zentralisierten Strukturen (Spitäler, Heime usw.) oder dezentral (Spitex der Gemeinde, «medical home», Versorgungsnetzwerk) in Zusammenarbeit mit Ärzten oder anderen Gesundheitsberufen tätig sein. Die Zusammenarbeit ist dabei auf eine optimale, zweckdienliche Betreuung und Begleitung von Patienten und ihren Angehörigen ausgerichtet. Pflegende übernehmen dabei je nach Situation koordinierende, leitende oder ausführende Funktionen. Sie übernehmen Aufgaben wie die Durchführung von Behandlungsmassnahmen nach ärztlicher Diagnosestellung und Therapiefestlegung, Vorsorgemassnahmen, Beratungen, Nachkontrollen und die langfristige Begleitung von Patienten.

Die Teamarbeit muss so organisiert sein, dass Pflegende sich auf das Wesentliche ihres Berufes konzentrieren können und von administrativen Aufgaben, Materialbewirtschaftung und Personalwesen entlastet sind.

Pflegende bieten ihre Dienstleistungen in vielen Fällen während 24 Stunden pro Tag und während 7 Tagen die Woche an. Die Rahmenbedingungen zur Ausübung des Berufes sind so zu gestalten, dass es den Pflegenden möglich ist, ihre Angebote wirkungsvoll, zweckmässig und wirtschaftlich selbstverantwortlich im Rahmen von beruflichen und fachlichen Standards anbieten zu können.

Voraussetzungen zur Ausübung des Berufes Eine professionell Pflegende bringt einen Berufsabschluss auf Bachelorstufe mit. Diese Ausbildung befähigt sie, den Beruf in seiner generellen Ausrichtung auszuüben. Danach kann eine Spezialisierung erfolgen.

Es werden Masterabschlüsse angeboten, die befähigen, in speziellen Arbeitsbereichen vertieftes Fachwissen/technische Fähigkeiten zu erwerben oder sich spezifischen Patientengruppen mit besonderem Beratungs- oder Begleitungsbedarf zuzuwenden. Pflegende mit einem Master können in diesen Fachbereichen eigenständig verantwortungsvolle Funktionen übernehmen.
Der Abschluss einer Weiterbildung befähigt die Pflegenden, in entsprechenden Fachbereichen, Tätigkeitsfeldern oder Techniken eigenverantwortlich tätig zu sein. Diese Weiterbildungen sind auf eine klar umschriebene berufliche Tätigkeit auszurichten. Standards und Qualitätsmerkmale sind dabei im Auge zu behalten. Ein modulares Weiterbildungssystem ermöglicht, sich im Laufe des beruflichen Werdegangs mit vertretbarem zeitlichem Aufwand weiterzuentwickeln, sich neu $\mathrm{zu}$ orientieren oder in ein benachbartes Berufsfeld zu wechseln. Durchlässigkeiten interdisziplinär und multidisziplinär sind eine Selbstverständlichkeit. Module sind mit anderen Gesundheitsberufen gemeinsam zu entwickeln und zu besuchen, wenn es sich um Grundlagen der Berufe im Gesundheitswesen handelt oder wenn es die interdisziplinäre oder multiprofessionelle Zusammenarbeit betrifft.

Pflegende mit einem Bachelor führen die Pflege praktisch durch, sie handeln dabei evidenzbasiert. Sie lernen im Laufe ihrer Ausbildung die wissenschaftlichen Methoden und Arbeitsweisen kennen. Ihre Mitarbeit bei Forschungsprojekten ist daher wünschbar. Studierende im Masterprogramm dagegen sind zwingend darauf angewiesen, Forschungsarbeiten unter Anleitung durchführen zu können. Eine eigenständige, wissenschaftliche Karriere erfolgt nach Erlangen eines Doktorats. Pflegende mit Masterausbildung können diesen Titel anstreben.

\section{Konflikte und Konsequenzen}

Auch angesichts aller Veränderungen der Gesellschaft, der beruflichen Tätigkeit und der Rahmenbedingungen muss das erste Ziel der Weiterentwicklung des Gesundheitswesens die optimale Patientenbetreuung sein und bleiben.

Die Veränderungen der Berufsbilder, wie wir sie skizzieren und erwarten, werden sich zum Teil unter dem Druck von Innovations- und Marktkräften von selbst ergeben.

Um sie in die Richtung zu lenken, die sich wirklich an den Bedürfnissen von Bevölkerung und Patienten orientiert, werden aber lenkende und unterstützende Massnahmen unumgänglich sein. Diese müssen vor allem in den Bereichen Aus- und Weiterbildung, Gestaltung innovativer Arbeitsmodelle, Honorierung und Gesetzgebung ansetzen.

Die zu erwartenden Entwicklungen bergen ein beträchtliches Konfliktpotential, wobei es vor allem zur Akzentuierung schon bestehender Konflikte und weniger zu völlig neuen Spannungsfeldern und Interessenkollisionen kommen dürfte. 
Es geht dabei vor allem um die Besitzstandswahrung und um die Schnittstellen zwischen Generalisten und Spezialisten, zwischen Ärzten, Pflegenden und anderen Berufen im Gesundheitswesen, zwischen dem ambulanten und stationären Sektor, zwischen freiberuflich Tätigen und Institutionen, zwischen dem öffentlichen und dem privaten Sektor und zwischen Leistungserbringern und Kostenträgern.

Falls es gelingt, zukunftsorientierte Berufsbilder sinnvoll umzusetzen und ihnen Akzeptanz zu vermitteln, wird sich die Zusammenarbeit verbessern, die Schnittstellenprobleme werden entschärft und die Effizienz der Leistungen verbessert. Vorerst aber werden einzelne Gruppen die traditionellen Rollen und Muster hartnäckig verteidigen, weil sie befürchten, dass gerade sie zu den Verlierern der Veränderungen gehören werden.

Ohne in die Details zu gehen, sieht die Arbeitsgruppe somit folgende Konsequenzen und Massnahmen, die mithelfen können, die potentiellen Konflikte zu entschärfen und den Wandel der Berufsbilder nicht einfach «geschehen zu lassen», sondern ihn, soweit überhaupt möglich, aktiv zu gestalten:

\section{Konsequenzen für die beiden Berufsgruppen der Ärzte und der Pflegenden}

- Die Kompetenzen und Verantwortlichkeiten von Ärzten und Pflegenden und diejenigen innerhalb der einzelnen Berufsgruppen sind neu zu definieren.

Für einen Teil dieser Abgrenzung können wohl allgemein akzeptierte Richtlinien erarbeitet werden, der andere Teil muss sinnvollerweise aber jeweils innerhalb der einzelnen Institutionen erarbeitet und festgelegt werden (Spital, Ambulatorium, Zentrum, Praxisgemeinschaft, Netzwerk). Für einzelne Arbeitsbereiche braucht es allerdings zuvor gesetzliche Anpassungen (z.B. für eine erweiterte Verordnungskompetenz für Pflegefachpersonen).

- Die Ausbildung auf Bachelorstufe vermittelt in Zukunft im Sinne eines «tronc commun» sowohl den Ärzten als auch den Pflegenden neben ihren jeweiligen spezifischen Kenntnissen gemeinsame Grundelemente (Prinzipien, Basiswissen, Fertigkeiten, Sprache; vgl. oben). Diese gemeinsamen Module müssen erarbeitet werden.

- Neue Modelle der interdisziplinären Teamarbeit (HMO, weiterentwickelte ManagedCare-Modelle, interdisziplinäre Praxisgemeinschaften, dezentrale Zusammenarbeit in Netzwerken usw.), bei der die einzelnen Berufe entsprechend ihren Kernkompetenzen ein- gesetzt werden können, sind zu fördern (vgl. oben). Staatliche Regulierungsmassnahmen und neue Versicherungsprodukte müssen unterstützend und nicht blockierend wirken.

Die Rahmenbedingungen sind so zu gestalten, dass es für Modelle mit Verbesserungen der interdisziplinären Zusammenarbeit möglichst wenig Schranken und keinen zusätzlichen administrativen Aufwand gibt.

- Die Berufsverbände haben die Aufgabe, die notwendigen Massnahmen einzuleiten und miteinander im konstruktiven Gespräch zu bleiben.

Der Geist des Beharrens und Bewahrens muss der Überzeugung Platz machen, dass im Gesundheitswesen neue Modelle und Konzepte aktiv gestaltet, getestet und immer wieder verbessert werden müssen.

\section{Konsequenzen für den Arztberuf}

- Die Lerninhalte auf Bachelor- und Masterstufe sind in regelmässigen Intervallen den gesellschaftlichen und fachlichen Erfordernissen anzupassen.

Die Ausbildung der Ärzte mit dem Berufsziel einer klinischen Tätigkeit bleibt auf Bachelorund Masterstufe im Kern für alle identisch. Für Studenten mit dem Berufsziel theoretische Medizin oder für ganz spezifische, technisch orientierte Berufe sind eigene Masterlehrgänge denkbar. Mit dem neuen Medizinalberufegesetz und der Bologna-Reform sind die Voraussetzungen geschaffen, um diese Anliegen umzusetzen.

- Für komplexe Gesundheitsstörungen sind Disease-Management-Modelle zu entwickeln und Patientenpfade in Diagnostik und Therapie zu definieren, für die jeweils ein generalistisch tätiger Arzt die Verantwortung übernimmt.

Diesem Arzt obliegt nach der Diagnosestellung primär die Aufgabe des Koordinators, er erklärt und bespricht mit den Patienten die sinnvollen Massnahmen. Durchgeführt werden diese Massnahmen jeweils durch die Fachpersonen innerhalb oder ausserhalb des Teams, die dafür geeignet und kompetent sind (z.B. Pflegefachperson, Assistentin, Spezialist, Spezialinstitution).

- Die Weiterbildung ist ab Beginn strukturiert und für alle Fachbereiche gleichwertig.

Klar definierte Curricula müssen den Anwärtern den Entscheid für ein Fachgebiet ermöglichen; ein solcher Entscheid ist mit Hilfe von Mentoring möglichst früh im Laufe der Weiterbildung zu treffen. 
- Es braucht genügend akkreditierte Weiterbildungsplätze in Spitälern und in Praxen und ein genügendes Angebot an Teilzeitstellen (v. a. auch für Frauen in Weiterbildung).

\section{Konsequenzen für Pflegeberufe}

- Der Bachelortitel muss auch in der Deutschschweiz und im Tessin zum Normalabschluss der Pflegeausbildung werden (in der Westschweiz seit 2002 eingeführt). Die Möglichkeiten, in der Pflege einen Master- oder Doktortitel zu erwerben, sind zu fördern.

- Die Lerninhalte sind im Hinblick auf den zukünftigen «tronc commun» in der Ausbildung mit den Ärzten anzupassen. Die Ausbildung auf Bachelorstufe vermittelt in Zukunft sowohl den Ärzten als auch den Pflegenden neben ihren spezifischen Kenntnissen gemeinsame Grundelemente (gleiche Module für Prinzipien, Basiswissen, Fertigkeiten, Sprache; vgl. oben).

- Die Nachwuchsrekrutierung ist mit Hinweis auf die breiten beruflichen Möglichkeiten zu intensivieren.

\section{Konsequenzen für die Politik, für die} Versicherer und für die Berufsorganisationen

- Die gesetzlichen Bestimmungen sind dahingehend zu überprüfen, dass ärztliche Delegationsregelungen und an institutionelle Gegebenheiten geknüpfte Tarifregelungen einer verbesserten Kompetenz- und Verantwortlichkeitsaufteilung nicht im Wege stehen. Gesetze und Verordnungen sind so anzupassen, dass die in diesem Bericht geforderte Anpassung der Kompetenzen und Verantwortlichkeiten möglich ist (z.B. Art. 25 Abs. 2a, Art. 35 KVG, Art. 7 KLV, Art. 49 KVV). Damit wird auch Pflegenden die Tätigkeit als selbständige Leistungserbringer zu Lasten der obligatorischen Krankenpflegeversicherung ermöglicht.

Art. 35 KVG führt ja jene Leistungserbringer auf, deren Leistungen von der obligatorischen Krankenpflegeversicherung bezahlt werden. In diese Liste sind auch selbständig tätige Pflegende aufzunehmen.

- Bestehende Tarifmodelle sind dahingehend anzupassen und zu flexibilisieren, dass die in diesem Bericht geforderte Neuverteilung von Kompetenzen und Verantwortlichkeiten die nötige finanzielle Grundlage findet.

Dies bedeutet zum Beispiel, dass bestimmte Leistungen pauschalisiert oder als Leistungsgruppe durch eine Gesundheitsorganisation verrechnet werden können.
- Netzwerkmodelle und die koordinierte Betreuung («coordinated care») müssen aktiv gefördert werden, damit die in diesem Bericht geforderte Neuverteilung von Kompetenzen und Verantwortlichkeiten auch in den Strukturen ihre Entsprechung findet.

Dies bedeutet, dass alle Stakeholder ihre Verantwortung gegenüber dem Gesundheitswesen wahrnehmen und innovative Versicherungsmodelle erarbeiten, die einerseits marktkonform sind, andererseits aber einer patientenzentrierten Optimierung der Leistungserbringung durch interdisziplinäre Teams nicht im Wege stehen.

- Die Berufsverbände sind gefordert, die unbedingt notwendige Informations- und Überzeugungsarbeit bei Politikern, Versicherern, Meinungsbildnern, anderen Entscheidungsträgern und in der Öffentlichkeit zu leisten. Erste Voraussetzung dafür ist, dass die eigenen Mitglieder hinter den prinzipiellen Überlegungen zu den Berufsbildern der Zukunft und hinter den auszuarbeitenden Detailkonzepten stehen.

\section{Empfehlung an den Vorstand der SAMW}

- Ausarbeitung einer «Charter for Coordinated Care»;

- Durchführung einer Veranstaltung zum Thema «Zukünftige Berufsbilder von Ärztinnen/Ärzten und Pflegenden» (Zielpublikum: Ärztinnen/Ärzte, Pflegende, Politiker, Versicherer, Journalisten).

Ausgewählte Literatur (in zeitlicher Reihenfolge)

- Kesselring A, de Geest S. Medizin und Pflege: siamesische Zwillinge? SAMWbulletin. 2002;(3):1-3.

- Bürgi H, Bader C, Bloch R, Bosman F, Horn B, Im Hof V, et al. Swiss Catalogue of Learning Objectives for Undergraduate Medical Training. Bern: SMIFK; 2002.

- SRK. Ausbildung zur dipl. Pflegefachfrau / zum dipl. Pflegefachmann. Ausbildungsbestimmungen. Bern: SRK; 2002.

- Kaufmann C. Medizin im Aufbruch: Neues Berufsbild, neues Gesetz, neue Ausbildung. www.unipublic. unizh.ch/magazin/gesundheit/2002/0595.

- Nordt C. Strukturwandel der medizinischen Grundversorgung: Ursachen und Wirkung der ärztlichen Arbeitszufriedenheit in unterschiedlichen Praxismodellen. Auszug (Zusammenfassung) aus Dissertation. Philosophische Fakultät, Universität Zürich; 2003.

- Charta zur ärztlichen Berufsethik. Schweiz Ärztezeitung. 2003;84(45):2347-9.

- Spichiger E, et al. Professionelle Pflege - neu definiert: Zwei Kernsätze und acht Ergänzungen. Krankenpflege. 2004;98(8):20-3. 
- Bovier P, Bouvier Gallacchi M, Goehring C, Künzi B. Santé des médecins de premier recours en Suisse. PrimaryCare. 2004;4(47):941-7.

- Buchan J, Calman L. Skill-Mix and Policy Change in the Health Workforce: Nurses in Advanced Roles. OECD Health Working Papers 17. Paris: OECD; 2005

- Gassmann B. Zusammenarbeit oder Konkurrenz? Schweiz Ärztezeitung. 2005;86(29/30):1763.

- Grüninger U. Braucht es den Hausarzt noch? PrimaryCare. 2005;5(3):58.

- Simoens S, Villeneuve M, Hurst J. Tackling Nurse Shortages in OECD Countries. OECD Health Working Papers 19. Paris: OECD; 2005.

- de Haller J. Gibt es bald keine Hausärzte mehr? (Interview). NZZ am Sonntag, 27.11.2005.

- Woodroffe E. Nurse-led general practice: the changing face of general practice? Br J Gen Pract. 2006; 56:632-3.

- Beer JH, Kaspar K. Ärztlicher Grundversorger als Berufsziel. NZZ, 14.3.2006.

- Barr M, Ginsburg J for the Health and Public Policy Committee of the American College of Physicians. The Advanced Medical Home. A Policy Monograph. Washington: ACP; 2006.
- Stiftung Careum (Hrsg.). Grenzen aufheben Thesen zur Zukunft der Ausbildung für Gesundheitsberufe. Zürich: Stiftung Careum; 2006.

- Freudenheim M. Attention Shoppers: Low Prices on Shots in Clinic. New York Times, 14.3.2006.

- Siegrist M, Orlow P, Giger M. Weiterbildung und Arbeitssituation aus Sicht der Assistenzärzte. Schweiz Ärztezeitung. 2006;87(10):379-86.

- Simoens S, Hurst J. The Supply of Physician Services in OECD Countries. OECD Health Working Papers 21. Paris: OECD; 2006.

- FMH. Ja zu Vernetzung und Qualität - ja zu «Managed Care». Thesen der FMH zu zwei aktuellen, zentralen Themen im Schweizer Gesundheitswesen. Schweiz Ärztezeitung. 2006;87(36):1535-9.

- Weinberger SE, Smith LG, Collier VU; Education Committee of the American College of Physicians. Redesigning training for internal medicine. Ann Intern Med. 2006;144(12):927-32.

- Sachverständigenrat zur Begutachtung der Entwicklung im Gesundheitswesen, D. Kooperation und Verantwortung. Voraussetzungen einer zielorientierten Gesundheitsversorgung. Kurzfassung, 106 Seiten. Bonn; 2007.

- SGIM. A Proposal for Coordinated Care. Policy Monograph. Basel: JGIM; 2007. 